\title{
Long-Term Coudé Radial-Velocity Studies With a 1.2-m Telescope
}

\author{
C.D. Scarfe \\ Department of Physics and Astronomy, University of Victoria, Victoria, \\ B.C., V8W 3P6, Canada
}

\begin{abstract}
I have used the $1.2 \mathrm{-m}$ telescope and coudé spectrograph of the Dominion Astrophysical Observatory for more than 30 years in a program of radial-velocity observations of binary stars. The program was begun with photographic plates as detectors, but for 20 years the primary detector has been the radial-velocity scanner, which cross-correlates stellar spectra with an artificial mask.

Since some of the binaries under observation have periods of several years, the instrument's stability is an important consideration. I have therefore been obliged to observe standard stars and asteroids to check its performance. These observations are of relevance to efforts to improve the IAU standard star system.

I will describe the telescope, spectrograph and scanner, and will briefly discuss some of the results obtained for a selection of binary and multiple stars.
\end{abstract}

\section{Introduction}

I have the good fortune to live only about $15 \mathrm{~km}$ from the Dominion Astrophysical Observatory, which forms the largest component of the National Research Council of Canada's Herzberg Institute of Astrophysics. I have thus had the opportunity to use that observatory's 1.2-m (48-inch) reflecting telescope and associated coudé spectrograph for most of those instruments' 38-year history, to pursue an observing program on binary and multiple stars. I have chosen to study systems many of which have periods of several years, which make it difficult to study them by infrequent visits to distant facilities.

In this paper I would like to outline the past history and present status of the telescope and the instruments I use with it, and to discuss a selection of the observations I have made.

\section{The 1.2-m Telescope}

The telescope was constructed by Grubb-Parsons and saw first light in 1962 . It is a standard off-axis equatorial, with a primary focal ratio $\mathrm{f} / 4$. The single pier and conveniently located counterweight make it easy to reach the pole, or to switch from side to side of the polar axis. The original primary mirror was replaced in 1985 by a low-thermal-expansion borosilicate glass one. The 
telescope is normally operated at the coude focus, using four additional mirrors to feed a horizontal spectrograph one floor below. In the late 1960's the original four mirrors were replaced by turrets of three mirrors each, with each of the three mirrors in each turret having a coating giving high reflectance in a specific wavelength region (Richardson 1968). They were necessarily small, and the beam between them is nearly parallel at $f / 145$. It is restored to $f / 30$ to match the spectrograph by a lens following the last mirror.

\section{The Coudé Spectrograph}

The spectrograph fills a heavily insulated room mounted on a concrete pad. Its collimator focal ratio is $\mathrm{f} / 30$, and it includes cameras of focal length $2.44 \mathrm{~m}$ and $0.81 \mathrm{~m}$ and gratings with 831,600 and 1200 rulings per mm. Four identical replica gratings with the first ruling are mounted together as a mosaic, used with the longer camera to give a reciprocal dispersion of $0.24 \mathrm{~nm} \mathrm{~mm}^{-1}$ in the second order, for which they are blazed at $400 \mathrm{~nm}$. The camera mirror is 0.91 $\mathrm{m}$ in diameter to avoid vignetting over a wavelength range of $70 \mathrm{~nm}$.

Light enters the spectrograph by way of a superpositioning image slicer (Richardson 1968). A cylindrical lens makes the beam slightly astigmatic so that it will pass first through the horizontal entrance slot of the image slicer and then through its vertical exit slit. The latter is narrow enough to provide good resolution, and significant amounts of light fall on its jaws rather than passing through. But that light may not be wasted; it is reflected back by small mirrors to another set of mirrors mounted inside the image slicer near the entrance aperture, from which it is reflected toward the slit again. The slicer is designed to distribute the light on the collimator so as to avoid losing light on the back of a plate holder or pick-off mirror. Thus not only does the slicer transmit more light for the same seeing conditions than does a conventional slit, but it also distributes that light somewhat better. The overall gain in speed over a slit is of the order of one magnitude.

The spectrograph was originally designed for use with photographic plates, but has been used with a Reticon detector, and more recently with a variety of CCD's. Experience with plates (Scarfe, Batten \& Fletcher 1990) showed that it has excellent stability for radial-velocity measurements, giving standard errors near $150 \mathrm{~m} \mathrm{~s}^{-1}$ for non-variable stars over many years. The velocities obtained from a carefully chosen set of reliable spectral lines have been found to agree well for bright asteroids with those predicted from their orbits (Scarfe 1985).

\section{The Radial-Velocity Scanner}

The scanner has been described by Fletcher et al. (1982) and McClure et al. (1985). It is based on a prototype built by Stilborn, Fletcher \& Hartwick (1972) following the concept of Griffin (1967), but it incorporates several unique features. Light passes through a mask modelled on the spectrum of a star, idealized so as to give full transmission in line regions and zero transmission in the continuum. The transmitted light is collected by a Fabry mirror system which forms an image of the mosaic grating on the cathode of an EMI 9835/350 photomultiplier tube. The cathode is of such small area $(9 \mathrm{~mm}$ by $9 \mathrm{~mm})$ that it does 
not require cooling to yield an acceptably low dark current, but to bring all the light to such a small area the Fabry mirror system is necessarily complex, as described by McClure et al. (1985).

The masks in regular use are based on the spectra of Arcturus (K2 III) and Procyon (F5 IV-V). They cover the spectral range $400-460 \mathrm{~nm}$. The Arcturus mask has about 750 slots each of which is $0.080 \mathrm{~mm}$ wide; those in the Procyon mask are slightly fewer and wider. The masks are scanned in dispersion by a stepping motor and the light transmitted is measured at each step and recorded automatically by a computer. For repeated scans the measurements at each step are binned in the computer so as to build up signal and average out the effects of seeing noise. The accumulated signal is displayed on an oscilloscope; the display is brought up to date after each scan, so that the observer can watch the progress of the observation and can terminate it when a satisfactory signal is built up. Drifts during the night are monitored by observations of an iron-argon hollow cathode lamp; the mask incorporates slots matching the lamp's spectrum.

The slots are arranged to make the spectral dispersion vary with height on the mask, and the mask is actually scanned at $45^{\circ}$ to the dispersion direction, so that the mask dispersion varies, as it must, as a function of the velocity being measured. In this way the mismatch discussed by Hearnshaw (1977) and Griffin (1977) is avoided, and velocities of several hundred $\mathrm{km} \mathrm{s}^{-1}$ can be measured without systematic error. It is of course necessary to adjust the zero point of the mask's height at the start of each night; this is readily done with the hollow cathode lamp, by covering alternately the red and blue halves of the mask, and ensuring that they give the same velocity.

Early experiments by McClure showed that the scanner's limiting magnitude could be increased by using an image slicer with an exceptionally wide exit slit, but at considerable cost in guiding error introduced to the radial velocities. This error was traced to systematically uneven illumination of the slit and collimator, and to avoid it a Guiding Error Minimizer (GEM) was introduced. This is a rotatable roofless Abbé prism placed in the beam just after the image slicer, which rotates the beam $180^{\circ}$ after every five scans. The computer control of the scanner was arranged to permit scanning to stop only after multiples of ten full scans whenever the GEM was in use. Guiding error is much less of a problem for the narrower image slicer that I normally use, but the GEM still gives a modest increase in the reliability of the data.

\section{Uncertainties of Measurement}

Experience has shown that the uncertainty of a measurement with the scanner is slightly less than $0.50 \mathrm{~km} \mathrm{~s}^{-1}$ for bright stars with sharp spectral lines, independent of spectral type, between F5 and M2. It is larger, however, for fainter objects and for those whose lines are weak, such as halo stars. Rapid rotation broadens the signal and increases the uncertainty. Indeed the minimum becomes almost impossible to detect for $v \sin i \geq 30 \mathrm{~km} \mathrm{~s}^{-1}$.

Part of the uncertainty may be ascribable to night-to-night variations in the instrument. But I have never been able to convince myself of the reality of any such effect; to do so would require an observer to devote a disproportionate amount of time to observations of standard stars. I have instead made a modest 
number of such observations - typically five per night, accumulating to about 100 or 150 per year - and have used them to determine the long-term observational uncertainties reliably instead. They have also provided reliable estimates of the zero-point differences between masks. These amount to as much as $1.25 \mathrm{~km}$ $\mathrm{s}^{-1}$ in extreme cases. I have also used some 150 observations of asteroids to determine the overall zero-point of the system (Scarfe 2000).

It should be noted that Skuljan, Hearnshaw \& Cottrell (1999) found significantly smaller uncertainties than those above from their observations with the same instrument. But their results are based on long runs of consecutive nights when the instrument was undisturbed, whereas mine are from many individual nights between which instrumental changes were made within the spectrograph. They also found a substantial year-to-year difference between runs; such differences may well be contributing to my larger uncertainties.

The instrumental profile of the scanner has maxima on either side of the central minimum, and shows additional, but smaller, fluctuations farther away in either direction. The maxima are due to the structure of the masks, which are opaque for a short distance on either side of each transparent region. This results in a complete mismatch on either side of the minimum, but random coincidences farther afield result in lower mean transmission there. The maxima and the unevenness, particularly the former, can cause systematic errors in the velocities measured for double-lined binaries, particularly for faint secondary stars. It is necessary to reduce traces of such objects more carefully than the usual method of fitting a parabola to the lower portion of the traces, which works well for single-lined objects. A program for such a reduction has been written by R.E.M. Griffin (Griffin 1991), who has kindly provided it for my use, for example, for the reduction of tracings of HR 6469 (Scarfe et al. 1994) as well as other similar systems.

\section{Some Illustrative Examples}

Several of the more interesting systems I have studied are triple. Triple systems usually contain a subsystem of short period and large velocity amplitude, which may permit us to avoid some of the blending of spectra that bedevils long-period binaries. But they often present complications of their own. We discuss three very different triple systems here, as examples.

\subsection{HR 6469}

This triple system, in which the distant component is an apparently spotted giant, was the subject of three consecutive papers in AJ (Wasson et al. 1994, Van Hamme et al. 1994, Scarfe et al. 1994). The close, eclipsing, main-sequence pair, whose combined mass is greater than that of the giant, was analysed photometrically in the second paper. The inclination of that pair, which is quite different from that found for the wide pair from speckle interferometry, allowed us to find all three masses, even though we could not detect the spectrum of the faintest component. We were able to find the distance to the system with an accuracy about $1.3 \%$, and reliable radii for the eclipsing components, but the masses are accurate only to about $5 \%$ for each star. Moreover, the largest contributor to these uncertainties is the amplitude of the motion, in the long-period 
orbit, of the centre of mass of the eclipsing pair, although the inclination of the long-period orbit also contributes substantially to the parallax uncertainty and to that of the combined mass of the eclipsing system and hence to the individual masses of its components. Nevertheless, this system did yield the mass of a giant star, reliable determinations of which remain scarce. And it provided luminosities and effective temperatures sufficiently reliable to make comparison with published evolutionary tracks worthwhile. That comparison in the end led us to a rough estimate of the system's age.

\subsection{HD 202908}

This system contains three similar main-sequence F-stars, and has been the subject of papers by Fekel (1981) and Fekel et al. (1997). A close, but apparently non-eclipsing pair in a four-day orbit has a distant companion moving around it in 78.5 years. The long-period system passed periastron in 1986, and was followed by no less than four independent radial-velocity observers and the CHARA speckle group, all of whose observations were pooled for the joint 1997 paper. In the end we managed to obtain a distance for the system accurate to better than $1.5 \%$, but the masses of the stars only to about $2.7 \%$. The principal source of the uncertainty in the distance is that of the angular major semiaxis, but that in the masses is derived predominantly from the velocity amplitudes in the long-period orbit. Once again, better interferometry and better radial velocities are both needed to reduce our uncertainty.

\subsection{Orionis (HR 2130)}

This system, consisting of three B-stars, was discussed by Fekel \& Scarfe (1986) and in more detail by Scarfe, Barlow \& Fekel (2000). Two sharp-lined objects form a pair with a period of two weeks, and a third body shares with them an orbit of period 13 years. This object is somewhat enigmatic in producing virtually no measurable spectral lines, despite contributing so much continuum light as to weaken the other components' lines considerably. Its luminosity is thus apparently comparable to that of either component of the short-period pair. The system has been resolved by a lunar occultation but is a difficult object for speckle interferometry. Our inability to measure the distant component's radial velocity means that we cannot determine the stars' masses without some assumption. However, the assumption that all three stars lie within the populated band of the mass-luminosity relationship defined by Andersen (1991) and Popper (1980) yields a strong constraint on the masses, and allows us to obtain them accurate to about $7 \%$. But new observations with very high signal-tonoise ratio will be needed, as well as additional interferometric observations of the wide pair, if this system is to provide us with the accurate B-star masses it seems capable of doing.

\section{Conclusions}

Many other objects are being followed in this program. Several new variables have been discovered among naked-eye stars. Patience is required, since among them are unresolved systems with periods in excess of ten years. Indeed a few have shown monotonic variations of radial velocity over intervals approaching 
two decades, and their periods are thus still unknown. Many of these systems should be targets for long-baseline interferometry, although some may prove in the end to have too large a magnitude difference for either interferometric resolution or spectroscopic detection of secondaries. But combination of these techniques should continue to yield accurate stellar masses, distances and luminosities. Such data are well worth pursuing.

Acknowledgments. I am most grateful to Murray Fletcher for correcting some factual errors in an earlier version of this paper. Any that remain are solely my own responsibility.

\section{References}

Andersen, J.A. 1991, A\&ARev 3, 91

Fekel, F.C. 1981, ApJ, 248, 670

Fekel, F.C., \& Scarfe, C.D. 1986, AJ, 92, 1162

Fekel, F.C., Scarfe, C.D., Barlow, D.J., Duquennoy, A., McAlister, H.A., Hartkopf, W.I., Mason, B.D., \& Tokovinin, A.A., 1997, AJ 113, 1095

Fletcher, J.M., Harris, H.C., McClure, R.D., \& Scarfe, C.D. 1982, PASP 562, 1017

Hearnshaw, J.B. 1977, Observatory 97, 5

Griffin, R.F. 1967, ApJ 148, 465

Griffin, R.F. 1977, Observatory 97, 9

Griffin, R.F. 1991, Observatory 111, 67

McClure, R.D., Fletcher, J.M., Grundmann, W.A., \& Richardson, E.H. 1985, in Stellar Radial Velocities (Proc. IAU Colloquium 88), ed. A.G.D. Philip \& D.W. Latham, L. Davis Pres, Schenectady, 49

Popper, D.M. 1980, ARA\&A 18, 115

Richardson, E.H. 1968, JRASC 62, 313

Scarfe, C.D. 1985, in Calibration of Fundamental Stellar Quantities (Proc. IAU Symposium 111), ed. D.S. Hayes, L.E. Pasinetti \& A.G.D. Philip, Dordrecht: Reidel, 583

Scarfe, C.D. 2000, Standard Star Newsletter 29, 10

Scarfe, C.D., Barlow, D.J., \& Fekel, F.C. 2000, AJ 119, 2415

Scarfe, C.D., Barlow, D.J., Fekel, F.C., Rees, R.F., McAlister, H.A., Hartkopf, W.I., Lyons, R.W., \& Bolton, C.T. 1994, AJ 107, 1529

Scarfe, C.D., Batten, A.H., \& Fletcher, J.M. 1990, Pub. DAO 18, 21

Skuljan, J., Hearnshaw, J.B., \& Cottrell, P.L. 1999, in Precise Stellar Radial Velocities (Proc. IAU Colloquium 170), ed. J.B. Hearnshaw \& C.D. Scarfe, ASP Conf. Ser. 185, 98

Stilborn, J.R., Fletcher, J.M., \& Hartwick, F.D.A. 1972, JRASC 66, 49

Van Hamme, W. et al. 1994, AJ, 107, 1521

Wasson, R. et al. 1994, AJ, 107, 1514 